\title{
POPULATION-BASED CASE-CONTROL INVESTIGATION OF RISK FACTORS FOR LEPTOSPIROSIS DURING AN URBAN EPIDEMIC
}

\author{
URMIMALA SARKAR, SIMONE F. NASCIMENTO, ROSAN BARBOSA, RIDALVA MARTINS, HECTOR NUEVO, \\ IPPOLYTOS KALAFANOS, ITAMAR GRUNSTEIN, BRENDAN FLANNERY, JUAREZ DIAS, LEE W. RILEY, \\ MITERMAYER G. REIS, AND ALBERT I. KO \\ School of Public Health, University of California, Berkeley, California; Gonçalo Moniz Research Center, Oswaldo Cruz \\ Foundation/Brazilian Ministry of Health, Salvador, Brazil; Department of Epidemiological Surveillance, Secretary of Health for the \\ State of Bahia, Salvador, Brazil; Division of International Medicine and Infectious Diseases, Weill Medical College of Cornell
} University, New York, New York

\begin{abstract}
Between March and October 2000, 157 suspected cases of leptospirosis hospitalized with complications of Weil's syndrome and a mortality of $8 \%$ were identified in Salvador, Brazil. We conducted a population-based casecontrol study to identify risk factors for acquisition of leptospirosis in neighborhoods with high endemicity during the rainy season-associated urban epidemic. Sixty-six (65\%) of 101 laboratory-confirmed cases and 125 age and sex-matched healthy neighborhood controls were interviewed. Residence in proximity to an open sewer (matched odds ratio [OR] $=$ $5.15,95 \%$ confidence interval $[\mathrm{CI}]=1.80-14.74)$, peri-domiciliary sighting of rats $(\mathrm{OR}=4.49,95 \% \mathrm{CI}=1.57-12.83)$, sighting groups of five or more rats $(\mathrm{OR}=3.90,95 \% \mathrm{CI}=1.35-11.27)$, and workplace exposure to contaminated environmental sources $(\mathrm{OR}=3.71,95 \% \mathrm{CI}=1.35-10.17)$ were found to be independent risk factors for acquiring disease. Some of these risk factors are amenable to focused interventions, which include provision of closed drainage systems for sewage and reduction of rodent populations in the peri-domicilary environment. Environmental control of transmission may help to greatly reduce the incidence of severe leptospirosis.
\end{abstract}

\section{INTRODUCTION}

Leptospirosis, which is caused by pathogenic spirochetes of the genus Leptospira, is acquired through contact with animal reservoirs or an environment contaminated by their urine. ${ }^{1-3}$ Although considered to be the most geographically widespread zoonosis, ${ }^{2,3}$ it has traditionally been a sporadic disease of rural and tropical settings, restricted to risk exposures associated with specific occupational groups, such as farmers, miners, and abattoir and sewer workers, ${ }^{1-3}$ and recreational activities. ${ }^{4,5}$ Recent outbreaks have raised new awareness concerning the risk of leptospirosis in disaster situations ${ }^{6,7}$ and sporting events. ${ }^{8,9}$

In contrast to these epidemiologic patterns, leptospirosis has been recognized as a major urban pathogen causing epidemics in Latin America. ${ }^{10-14}$ In Brazil alone, approximately 10,000 cases are reported each year from all major cities (case notification records, Epidemiological Surveillance Department, Brazilian Ministry of Health). Cases have severe clinical presentations such as Weil's syndrome ${ }^{14,15}$ and severe pulmonary hemorrhage syndrome. ${ }^{16-18}$ Overall mortality is $10-15 \%$ among outbreak cases. ${ }^{14,15}$ For those who develop severe pulmonary hemorrhage syndrome, mortality exceeds $50 \% .{ }^{17}$

Lack of basic sanitation in urban slums (favelas), together with frequently contaminated environmental exposures during seasonal heavy rainfall and flooding, is speculated to contribute to annual epidemics. ${ }^{14,19,20}$ Rattus species are presumably the primary reservoirs, as observed during sporadic transmission in inner cities in the United States. ${ }^{21-24}$ However, beyond the widespread phenomena of climate and poverty, specific risk exposures at the community level have not been addressed. We previously described a large epidemic in 1996 associated with severe forms of leptospirosis and high mortality in the city of Salvador, Brazil. ${ }^{14}$ In this study, we present findings of a population-based matched case-control investigation, performed during a subsequent outbreak in 2000 , to identify risk factors for acquisition of urban leptospirosis.

\section{MATERIALS AND METHODS}

Surveillance site. During the period of heavy seasonal rainfall between March and October 2000, an outbreak of leptospirosis was identified in Salvador, a coastal city with more than two million inhabitants in northeastern Brazil, through active surveillance based at a 120-bed, state-run infectious disease referral hospital. According to state health secretary protocol, suspected cases of leptospirosis from the metropolitan area are referred to this hospital. Notification of leptospirosis cases to the state health secretary is mandatory and the surveillance hospital was the source of $98 \%$ of the cases reported during the outbreak period.

Case definition. Patients with leptospirosis were identified according to a surveillance case definition based on the presence of conjunctival suffusion, a specific finding on physical examination for leptospirosis, ${ }^{3}$ jaundice and acute renal failure (oliguria defined as urine output $<500 \mathrm{ml} / 24 \mathrm{~h}$, serum creatinine $>2.0 \mathrm{mg} / \mathrm{dL}$, or blood urea nitrogen $>150 \mathrm{mg} / \mathrm{dL}$ ). Patients were excluded if they had radiologic or laboratory evidence for another disease during hospitalization or had a discharge diagnosis other than leptospirosis. Informed consent was obtained from patients or their guardians according to the guidelines for conduct of the research with human subjects of the institutional review boards of Brazilian Ministry of Health, the New York-Presbyterian Hospital, and the University of California at Berkeley. Demographic data, clinical history, and laboratory findings were obtained through interview and medical chart review for all patients meeting the surveillance case definition.

Selection of cases and controls. For the case-control study, cases were randomly selected from patients who were hospitalized between March 8, 2000 and October 31, 2000, met the surveillance definition of leptospirosis, were greater than 12 years of age, and had a positive anti-leptospiral IgM enzymelinked immunosorbent assay (ELISA) reaction in one or more serum samples. Residential addresses were used to locate cases. For each case patient who could be located and 
agreed to participate in the study, two age and sex-matched controls were identified in the same neighborhood as that of the cases. Sex-specific age quartiles were pre-determined based on 1996-1999 surveillance data for Salvador and used to match control subjects with cases. Immediately after interview of the case patient, the field team surveyed households a distance of five domiciles from the case household, and at every household thereafter, to identify an appropriate age and sex-matched control subject. The second control subject was located in a similar manner, starting five domiciles from the household of the first control subject. Control individuals lived within 50 meters of cases with first control subjects located 5-10 domiciles from the case residence and second control domiciles located $10-18$ residences from the case residence. Potential control individuals who recalled receiving the diagnosis of leptospirosis in the previous year were excluded. Cases and control subjects were enrolled into the study according to approved informed consent procedures.

Data collection and definitions. The study team administered a standardized questionnaire during interviews conducted between October 2000 and March 2001. The questionnaire examined potential risk factors associated with demographic and socioeconomic data; residential sanitary conditions; work-related, recreational, and domestic activities; and exposure to contaminated environmental sources and potential animal reservoirs. The data collected were based on interview responses and visual inspection for the presence of an open sewer and accumulated refuse at the residence. The following definitions were used to code responses. "Work" was defined as formal or informal-sector remunerative activity taking place within the last six months. "Proximity to an open sewer" was defined as residing within five meters of a collection or outflow of residential wastewater with inadequate cement covering. Similarly, the continuous presence of household trash within five meters of a residence was defined as "proximity to accumulated trash." Visual sighting of rats by study subjects was used to assess presence of rodent reservoirs. Subjects were further asked to specify the location of rat sighting (inside the home, on the porch, on the street, at work, other) and the number of rats sighted at a time (one rat, two to four rats, five or more rats.) Identification of rats in the peri-domiciliary setting was defined as sighting rats on the porch adjoining the subject's residence.

Serologic case confirmation. As part of the surveillance protocol, paired acute and convalescent-phase serum samples were obtained from suspected patients within 24 hours of admission and at a follow-up outpatient visit after hospital discharge ( $>14$ days after collection of the first sample), respectively. Samples were tested in an anti-leptospiral IgM ELISA according to the method of Terpstra and others ${ }^{25}$ and Adler and others ${ }^{26}$, with the modification that a strain of Leptospira interrogans serovar copenhageni, isolated from a patient in Salvador, ${ }^{14}$ was used as the antigen source. The cut-off for seropositivity was defined as the absorbance value of the 96th percentile in serum samples obtained during a city-wide seroprevalence survey of healthy individuals. For hospitalized patients with leptospirosis, the sensitivity was $70 \%$ and $100 \%$, respectively, during the acute and convalescent phases of the illness. Cases were defined as confirmed if an acute or convalescent serum sample had absorbance greater than a threshold optical density used to define a positive reaction. ${ }^{26}$

At the time of interview, a convalescent-phase serum sample was collected from cases that did not previously submit a sample. All cases included in the case-control study had a positive IgM ELISA result in either the acute or convalescent-phase serum sample. As an additional confirmatory test, the microagglutination test (MAT) was performed as previously described ${ }^{14} \mathrm{~A}$ case was MAT-confirmed if there was a four-fold increase in agglutination titers between paired samples, a reciprocal titer $>800$ in one or both samples, or seroconversion from no titer $(<100)$ in the acute phase to a reciprocal titer $\geq 400$ in the convalescent phase. Cases and their matched controls were excluded from data analysis if the case did not have a MAT-confirmed diagnosis. A blood sample was collected from neighborhood controls subjects at the time of interview for the assessment of a recent exposure to leptospirosis. Control subjects were excluded from the matched pair during analysis if they had a positive IgM ELISA result.

Statistical analysis. Epi-Info (version 6.04) software was used for data entry and analysis from hospital-based surveillance and the case-control study. Analysis of variance and the chi-square test were used to compare means and proportions, respectively. The association of risk factors with the acquisition of leptospirosis was first assessed by univariate analyses. Continuous variables were dichotomized at the median value unless otherwise noted. Mantel-Haenzel odds ratios (ORs) for matched data and summary $\chi^{2}$ with the McNemar correction were used to assess univariate associations. Highly correlated covariates, such as work-related exposures to different types of environmental sources of contamination, were combined into a single variable for multivariate analysis. A backward-elimination method was used to test all variables significant in univariate analysis in a conditional logistic regression model. Multivariate analysis was performed with Intercooled STATA 6.0.

\section{RESULTS}

Outbreak investigation. Between March 8 and October 31, 2000, a period that includes the peak rainfall season, 157 patients were hospitalized who met the surveillance criteria for leptospirosis and who were residents of urban slums within the city of Salvador. Among these, 13 (8\%) died during hospitalization and all identified cases had a clinical diagnosis of leptospirosis on hospital discharge or death. Of the 157 cases, $101(64 \%)$ had serologic evidence of leptospirosis in the IgM ELISA. For patients with paired serum samples, the positive predictive value of the surveillance case definition was $94 \%$ (101 of 108). Seven patients did not have serologic evidence of leptospirosis who met the surveillance definition and for whom paired serum specimens were available. Based on the 150 hospitalized cases after excluding those who did not have serologic evidence of leptospirosis after testing paired serum samples, the incidence of severe leptospirosis for the city of Salvador (population $=2,211,539$ inhabitants $^{27}$ ) was 6.8 per 100,000 persons during the study period.

Among the 101 confirmed cases, 90\% were males. The mean \pm SD age was $35.0 \pm 14.2$ years for males and $43.3 \pm 12.4$ years for females (Table 1). Severe clinical manifestations identified during hospitalization included jaundice (89\%) and acute renal insufficiency (serum creatinine $>2.0 \mathrm{mg} / \mathrm{dL}, 75 \%$; 
TABLE 1

Demographic and clinical characteristics of leptospirosis patients identified through active surveillance

\begin{tabular}{|c|c|c|c|c|}
\hline \multirow[b]{2}{*}{ Characteristics } & \multicolumn{2}{|c|}{ Selected cases $(n=66)$} & \multicolumn{2}{|c|}{ All confirmed cases* $(\mathrm{n}=101)$} \\
\hline & Responses & $\begin{array}{c}\text { No. (\%) } \\
\text { or mean (SD) }\end{array}$ & Responses & $\begin{array}{c}\text { No. (\%) } \\
\text { or mean (SD) }\end{array}$ \\
\hline \multicolumn{5}{|l|}{ Demographics } \\
\hline Age & 66 & $35.2(14.8)$ & 101 & $36.0(14.2)$ \\
\hline Male gender & 66 & $58 \quad(88)$ & 101 & $90 \quad(89)$ \\
\hline \multicolumn{5}{|l|}{ Clinical presentation } \\
\hline Jaundice & 66 & $57 \quad(86)$ & 101 & $90 \quad(89)$ \\
\hline Total serum bilirubin $(\mathrm{mg} / \mathrm{dL})$ & 66 & $17.0(14.6)$ & 82 & $16.7(14.0)$ \\
\hline Serum creatinine $(\mathrm{mg} / \mathrm{dL})$ & 66 & $4.0(2.3)$ & 100 & $4.3(2.7)$ \\
\hline Blood urea nitrogen $(\mathrm{mg} / \mathrm{dL})$ & 66 & $193.5(130.0)$ & 100 & $199.1(143.5)$ \\
\hline \multicolumn{5}{|l|}{ Outcomes } \\
\hline Intensive care unit admission & 66 & 11 (17) & 101 & $19 \quad(19)$ \\
\hline Days of hospitalization & 66 & $8.8(6.5)$ & 101 & $8.9(45.2)$ \\
\hline Mortality rate & 66 & $0 \quad(0)$ & 101 & 2 (2) \\
\hline
\end{tabular}

* Patients residing in the city of Salvador, Brazil who were hospitalized between March 8 and October 31, 2000 with initial and final diagnosis of leptospirosis and laboratory evidence (IgM enzyme-linked immunosorbent assay) of infection in one or more serum samples.

blood urea nitrogen $>100 \mathrm{mg} / \mathrm{dL}, 68 \%$ ). Intensive care monitoring and intraperitoneal dialysis was required in $19(19 \%)$ and $21(21 \%)$, respectively. The mortality rate was $2 \%$ among confirmed cases.

Case-control study. Of the 101 patients with laboratory confirmation of leptospirosis, 83 (82\%) were selected randomly as cases for the case-control study. Of these 83 patients, $66(80 \%)$ cases were located at their residence and agreed to participate in the study. All 66 cases had serologic evidence of leptospirosis according to MAT and IgM ELISA criteria. Cases that were selected did not differ significantly from those who were not selected with respect to age, sex, and clinical presentation and outcome (Table 1).

We identified 134 individuals who were matched by age, sex, and neighborhood of residence to cases. Of these, 132 (99\%) agreed to participate in the study and were interviewed. Serum samples were obtained from 103 (78\%) control subjects. Among these, seven (7\%) who had documented evidence for recent exposure to leptospirosis according to the IgM ELISA were excluded from the analyses. The 66 cases and 125 control individuals had incomes within national poverty levels: for the 191 study subjects, median monthly personal and per capita household incomes were US\$75 and US\$44, respectively.

Table 2 summarizes the findings of matched univariate analyses for risk factors associated with the acquisition of leptospirosis. There were no significant differences between cases and control subjects with respect to race $(95 \%$ versus $90 \%$, respectively, which were black or mixed race) and mean personal income ( $\$ 120.90$ and $\$ 121.70$, respectively). The mean number of residents was 5.3 for household of cases and 4.7 for those for controls subjects $(P>0.05)$ and mean monthly household income did not differ significantly between cases and control subjects (US\$59.25 versus $\$ 65.15$ per capita, respectively). Leptospirosis was not associated with a residence of less than two years in the neighborhood, a history of leptospirosis, or a household member who had leptospirosis $(P>0.05)$.

Residential sanitary conditions. Proximity to an open sewer was significantly associated with acquiring leptospirosis (matched OR $=5.07,95 \%$ CI $=2.04-12.64): 42$ (64\%) of 66 cases had an open sewer within five meters of their residence compared with 48 (38\%) of 125 control individuals (Table 2). Cases also reported flooding in front of their residence more frequently than did control subjects (44\% versus $30 \%$; matched OR $=2.54,95 \% \mathrm{CI}=1.08-6.17)$. In contrast, access to potable water, peri-domiciliary trash accumulation, and municipal waste collection were not found to be significant risk factors. Visual inspection of accumulated trash and open sewer proximity was used to confirm subject responses for these exposures. Matched analyses did not identify a significant risk associated with specific peri-domiciliary (household cleaning and repair, food preparation, washing clothes), recreational, or leisure activities.

Environmental sources of exposure. Cases and control subjects had frequent contact with environmental sources of contamination; $83 \%$ and $60 \%$, respectively, reported contact with sewage, floodwater, or mud during daily activities. The daily time periods spent outdoors ( $>6$ hours) and outside the domicile ( $>6$ hours) were associated with an increased risk for acquiring leptospirosis (matched $\mathrm{OR}=2.67,95 \% \mathrm{CI}=1.03$ 7.64 and matched $\mathrm{OR}=3.00,95 \% \mathrm{CI}=1.35-6.75$, respectively). However, contact with any one of these sources was associated with increased risk of acquiring leptospirosis in the matched analysis (Table 2). For the 55 cases that reported contact with potential sources of contamination, $46(84 \%)$ reported exposure in the street, $31(56 \%)$ at the workplace, and $20(36 \%)$ in the household setting.

Work-related activities. Among the 191 study subjects, $80 \%$ (141) received remuneration for work activities and $38 \%$ (72) had multiple work activities through informal employment. Cases and control subjects were engaged in manual labor activities such as construction (35 versus $23 \%$, respectively), outdoor vending (9 versus $3 \%$ ), and domestic service (5 versus $4 \%)$. A higher, although not significant $(P=0.077)$, proportion of cases worked as mechanics when compared with control subjects ( 9 versus $2 \%$; matched OR $=10.00$; $95 \%$ CI $=1.00-15.99$ ). Furthermore, working in an outdoor environment and workplace exposure to sewage, floodwater, or mud were associated with increased risk for leptospirosis (matched OR $=2.46,95 \% \mathrm{CI}=1.04-5.11$ and matched OR $=3.27,95 \% \mathrm{CI}=1.48-7.22$; respectively).

Reservoirs. Both cases and control subjects lived in a ratinfested environment: $92 \%$ and $88 \%$, respectively, reported 
TABLE 2

Odds ratios and $95 \%$ confidence intervals for risk factors for acquiring leptospirosis during urban epidemics in a matched univariate analysis

\begin{tabular}{|c|c|c|c|c|}
\hline Variable & $\begin{array}{c}\text { Cases [No. }(\%)] \\
(\mathrm{n}=66)\end{array}$ & $\begin{array}{l}\text { Control individuals } \\
{[\text { No. (\%) }} \\
(\mathrm{n}=125)\end{array}$ & OR $(95 \% \mathrm{CI})^{*}$ & $P^{\dagger}$ \\
\hline \multicolumn{5}{|l|}{ Residental sanitary conditions } \\
\hline Open sewer in proximity & $42(64)$ & $48(38)$ & $5.07(2.04-12.64)$ & $<0.001$ \\
\hline Open sewer floods during rain season & $25(38)$ & $25(20)$ & $4.21(1.51-12.83)$ & $<0.001$ \\
\hline Street floods during rain season & $29(44)$ & $37(30)$ & $2.54(1.08-6.17)$ & $<0.05$ \\
\hline Accumulated refuse & $24(36)$ & $36(29)$ & $1.53(0.70-3.31)$ & \\
\hline No refuse collection service & $8(12)$ & $14(11)$ & $1.15(0.26-4.71)$ & \\
\hline \multicolumn{5}{|l|}{ Exposure to contaminated sources } \\
\hline$>6 \mathrm{~h} /$ day outdoors & $41(62)$ & $56(45)$ & $2.42(1.16-5.00)$ & $<0.05$ \\
\hline Contact with sewer water & $33(50)$ & $27(22)$ & $3.63(1.69-7.25)$ & $<0.001$ \\
\hline Contact with floodwater & $44(67)$ & $58(46)$ & $3.03(1.44-6.39)$ & $<0.001$ \\
\hline Contact with mud & $34(52)$ & $37(30)$ & $3.08(1.32-5.87)$ & $<0.005$ \\
\hline \multicolumn{5}{|l|}{ Reservoirs } \\
\hline Sighting rats & $61(92)$ & $110(88)$ & $1.81(0.57-6.57)$ & \\
\hline Sighting groups of five or more rats & $27(41)$ & $22(18)$ & $5.00(2.22-21.25)$ & $<0.001$ \\
\hline Peri-domiciliar sighting of rats & $46(70)$ & $58(46)$ & $3.40(1.74-11.78)$ & $<0.001$ \\
\hline Sighting rats in the street & $54(82)$ & $98(78)$ & $1.30(0.56-3.15)$ & \\
\hline Sighting rats at work site & $25(38)$ & $27(22)$ & $2.40(1.11-5.17)$ & $<0.05$ \\
\hline Cat at residence & $10(15)$ & $18(14)$ & $1.03(0.37-2.66)$ & \\
\hline Dog as domestic animal & $25(38)$ & $43(34)$ & $1.19(0.57-2.47)$ & \\
\hline \multicolumn{5}{|l|}{ Work-related activities } \\
\hline Works > 40 h/week & $39(59)$ & $59(47)$ & $1.72(0.89-3.66)$ & \\
\hline Works outdoors exclusively & $35(53)$ & $44(35)$ & $2.46(1.04-5.11)$ & $<0.05$ \\
\hline Work-related contact with trash & $30(45)$ & $34(27)$ & $2.36(1.23-5.56)$ & $<0.01$ \\
\hline Use of gloves during work & $21(32)$ & $25(20)$ & $1.89(0.91-4.00)$ & \\
\hline \multicolumn{5}{|l|}{ Occupation } \\
\hline Sewer worker & $13(20)$ & $12(10)$ & $2.25(0.89-7.04)$ & \\
\hline Construction worker & $20(30)$ & $37(30)$ & 1.09 (0.51-2.27) & \\
\hline Mechanic & $6(11)$ & $3(2)$ & $10.00(1.00-15.99)$ & \\
\hline Refuse disposal worker & $9(14)$ & $9(7)$ & $1.94(0.68-6.08)$ & \\
\hline
\end{tabular}

$\dagger$ The McNemar test was used to calculate $P$ values. Values are not shown for nonsignificant associations $(P \geq 0.05)$ for the purpose of clarity.

sighting rats during their daily activities. However, more specific questions revealed differences in rat sightings between cases and controls. A higher proportion of cases reported sighting five or more rats compared with control subjects $(41 \%$ versus $18 \%$; matched $\mathrm{OR}=5.00,95 \% \mathrm{CI}=2.22$ 21.25). In addition, sighting rats in peri-domiciliary (matched $\mathrm{OR}=3.40,95 \% \mathrm{CI}=1.74-11.78)$ or workplace settings (matched OR $=2.40,95 \% \mathrm{CI}=1.11-5.17$ ) were significant risk factors for leptospirosis, whereas sighting rats in the street was not $(P<0.05)$. The presence of a cat in the household did not significantly confer protection against acquiring leptospirosis. In contrast to association with exposure to rats, contact with dogs, mice, pigs, sheep, and other potential reservoirs in the workplace and household setting or during rec- reational activities was not significantly associated with disease acquisition.

Multivariate analysis. Table 3 shows the independent risk factors identified in the conditional logistic regression model. Residential proximity to an open sewer had the strongest association with acquisition of leptospirosis in the multivariate model (matched OR $=5.15,95 \% \mathrm{CI}=1.80-14.74$ ). Sighting more than five rats as an indicator of rat density (matched OR $=3.90,95 \% \mathrm{CI}=1.35-11.27)$, peridomiciliary rat infestation (matched OR $=4.49,95 \% \mathrm{CI}=$ 1.57-12.83) and occupational contact to sewer water, floodwater, or mud (matched OR $=3.71,95 \% \mathrm{CI}=1.35-10.17$ ) were additional independent risk factors for acquiring leptospirosis. Among all cases, $95 \%$ reported at least one of the

TABLE 3

Unadjusted and adjusted odds ratios and 95\% confidence intervals risk factors for acquisition of leptospirosis

\begin{tabular}{|c|c|c|c|c|}
\hline Risk factor & $\begin{array}{c}\text { Cases (No. }(\%)) \\
(\mathrm{n}=66)\end{array}$ & $\begin{array}{l}\text { Control individuals } \\
\text { (No. }(\%)) \\
(\mathrm{n}=125)\end{array}$ & Unadjusted $\mathrm{OR}(95 \% \mathrm{CI})^{*}$ & Adjusted OR $(95 \% \mathrm{CI}) \dagger$ \\
\hline Open sewer in proximity to residence & $42(64)$ & $48(38)$ & $5.08(2.04-12.64)$ & $5.15(1.80-14.74)$ \\
\hline Peridomiciliar sighting of rats $\ddagger$ & $46(70)$ & $58(46)$ & $3.40(1.74-11.78)$ & $4.49(1.57-12.83)$ \\
\hline Sighting groups of five or more rats & $27(41)$ & $22(18)$ & $5.00(2.22-21.25)$ & $3.90(1.35-11.27)$ \\
\hline Workplace exposure to contamination§ & $35(53)$ & $31(25)$ & $3.27(1.48-7.22)$ & $3.71(1.35-10.17)$ \\
\hline
\end{tabular}

* Mantel-Haentzel odds ratios (ORs) and exact $95 \%$ confidence intervals (CIs) comparing cases and controls matched by age, sex and neighborhood.

$\dagger$ Odds ratio from conditional logistic regression with matched data adjusted for the other risk factors in the model.

$\doteqdot$ Defined as sighting of rats on the porch of the subject's residence.

$\S$ Defined as contact with floodwater, sewer water, or mud at workplace. 
independent exposures compared with $75 \%$ of the control subjects.

\section{DISCUSSION}

In this population-based, case-control investigation of a leptospirosis epidemic in the city of Salvador, Brazil, residence in proximity to an open sewer, peri-domiciliary rat sightings, sighting of five or more rats, and workplace exposure to contaminated water or soil were identified as significant risk factors for acquisition of severe leptospirosis. Residing in proximity to an open sewer was the strongest factor associated with illness in the logistic regression model (matched OR $=5.50)$, despite the fact that control subjects were selected from the same neighborhoods. Therefore, even within a neighborhood lacking basic sanitation, sampling within small distances allowed differences in sewer-related exposures to be detected. Similarly, although $90 \%$ of all respondents reported sighting rats, sighting five or more rats was independently associated with disease. Together, these findings suggest potential dose-related differences in exposure between cases and control individuals.

Exposure to flood or sewer water and mud at the workplace was also a risk factor for acquiring leptospirosis. However, in Salvador, the workplace for informal employment is often located within the same areas where the study subjects reside, making it difficult to separate workplace exposures from those associated with the peri-domiciliary environment. Identification of residential proximity to an open sewer and household rat infestation as risk factors indicates that much of urban leptospirosis during the epidemic season may be acquired through peri-domiciliary transmission.

Furthermore, the findings from this study provide epidemiologic evidence supporting the role of domestic rats as the principal reservoir during urban epidemics. More than $90 \%$ of the serovars isolated from patients during urban epidemics in Brazil belong to Icterohaemorrhagiae, ${ }^{14,28}$ a serogroup commonly associated with Rattus species reservoirs. ${ }^{1,3}$ Sighting five or more rats and peri-domicilary rat sightings were independent risk factors for leptospirosis, whereas reported contact with other potential reservoirs, including mice and dogs, was not observed to be significantly associated with disease acquisition. Cases did not report direct contact with rats, suggesting that leptospiral transmission appears to occur primarily through exposure to an environment contaminated by the urine of rodent reservoirs.

The study was limited by the case-identification protocol based on a sample of cases with severe leptospirosis hospitalized during one epidemic season. Risk exposures associated with acquisition of milder forms of leptospirosis may not necessarily be the same as those that were identified to be associated with severe disease forms such as Weil's syndrome. Among 101 laboratory-confirmed patients who met the surveillance definition, $20 \%$ were not investigated because of death, inability to locate due to change of residence, or the random selection method used. To compensate for the sample size, two control subjects were matched to each case. In addition, matching according to neighborhood may have inadvertently masked other risk factors. Furthermore, as with all case-control outbreak investigations, the ability to recall exposures could have differed between case and control individuals. For example, exposure to pathogenic leptospires presumably occurs during peak periods of rainfall, ${ }^{14,29}$ and these time-related exposures could not be evaluated in this study due to limitations in subject recall.

Although annual rainfall-associated epidemics of leptospirosis in urban settings have long been recognized in Brazil, ${ }^{10-12}$ specific risk factors during such epidemic periods have not been previously identified. Due to urban population growth and the changing pattern of poverty in the last 40 years, ${ }^{30}$ a large proportion of the population in Latin America now resides in urban slums. Ecologic studies of urban epidemics of leptospirosis identified that cases geographically clustered in these areas of poor sanitation and flooding during periods of heavy rainfall. ${ }^{14,19,20}$ The observations from this case-control investigation show that there are definable peridomiciliary infrastructure deficiencies within urban slums that contribute to these outbreaks. These identified risk factors are conditions that are potentially correctable not by individuals, but at the municipal and community level.

The severe clinical outcomes of leptospirosis drain developing-country health resources. During this single outbreak in the city of Salvador, more than 150 cases were hospitalized with complications of jaundice, acute renal failure, and bleeding diatheses necessitating intensive care monitoring and dialysis. The overall mortality rate was $8 \%$. In other cities such as Rio de Janeiro and São Paulo, outbreaks of leptospirosisassociated severe pulmonary hemorrhage syndrome are associated with mortality greater that $50 \% .^{17,18}$ Moreover, cases of severe leptospirosis identified by hospital-based surveillance are only a small fraction of all leptospiral infections. Severe disease is believed to represents $5-15 \%$ of clinical infections; ${ }^{1,3}$ therefore, there may have been as many as 3,000 cases associated with this single outbreak. Furthermore, 7\% of control subjects had anti-leptospiral IgM antibodies, indicating that a significant proportion of the population residing in high-risk regions are exposed to leptospires and may have developed recent sub-clinical or asymptomatic infections.

Because urban epidemics of leptospirosis are characterized by high mortality within the first 48 hours of hospitalization, ${ }^{14}$ prevention and improved detection are required to improve clinical outcomes at the population level. Clearly, urban slums require adequate sewage containment and treatment urgently. In addition, rodent control programs must be targeted to decrease rodent populations in the peri-domiciliary and workplace environment in anticipation of annual periods of heavy rainfall. Finally, programs to limit exposure to pathogenic leptospires must extend to occupational settings. Further study is required to define and prevent exposures with contaminated water and soil in situations of informal employment and work conditions associated with urban poverty.

Acknowledgments: We thank Fernanda Albuquerque and Adriana Almeida for their assistance with the laboratory diagnostic evaluation, Amaro Nunes da Silva for locating patients, and Avani Marise Gomes for administrative assistance.

Financial support: This work was supported by funding from the Oswaldo Cruz Foundation, Brazilian Ministry of Health (0250.250.415); the Brazilian National Research Council (52.1229/ 98-7 and 350.052/95-6, 300.861/96-6 and PRONEX 4196086200); the Fogarty International Center; and the National Institutes of Health (Bethesda, MD) (Public Health Service Grants AI-01605, TW-00905, and TW-00919).

Authors' addresses: Urmimala Sarkar, Ippolytos Kalafanos, Itamar Grunstein, Brendan Flannery, and Lee W. Riley, School of Public 
Health, University of California, 140 Warren Hall, Berkeley, CA 94720-7360, Telephone: 510-643-2949, Fax: 510-642-6350. Simone F. Nascimento, Rosan Barbosa, Ridalva Martins, Hector Nuevo, and Mitermayer G. Reis, Centro de Pesquisas Gonçalo Moniz; Fundação Oswaldo Cruz/MS, Rua Waldemar Falcão, 121; 40295-001 Salvador, Bahia, Brazil, Telephone: 55-71-356-4320 ext 243, Fax: 55-71-3562155. Juarez Dias, Departamento de Vigilância Epidemiológica, Secretária da Saúde do Estado da Bahia, Centro Administrativo, 40000 Salvador, Bahia, Brazil, Telephone: 55-71-336-6419, Fax: 55-71-3710655. Albert I. Ko, Centro de Pesquisas Gonçalo Moniz; Fundação Oswaldo Cruz/MS, Rua Waldemar Falcão, 121; 40295-001 Salvador, Bahia, Brazil, Telephone: 55-71-356-4320 extension 243, Fax: 55-71356-2155 and Division of International Medicine and Infectious Diseases, Weill Medical College of Cornell University, 1300 York Ave, A-431, New York 10021, Telephone: 212-746-6320; Fax: 212-7468675, E-mail: aik2001@med.cornell.edu.

Reprint requests: Albert I. Ko, Centro de Pesquisas Gonçalo Moniz; Fundação Oswaldo Cruz/MS, Rua Waldemar Falcão, 121; 40295-001 Salvador, Bahia, Brazil, Telephone: 55-71-356-4320 extension 243, Fax: 55-71-356-2155, E-mail: aik2001@med.cornell.edu.

\section{REFERENCES}

1. Farr RW, 1995. Leptospirosis. Clin Infect Dis 21: 1-6.

2. Levett PN, 2001. Leptospirosis. Clin Microbiol Rev 14: 296-326.

3. Faine SB, Adler B, Bolin C, Perolat P, 1999. Leptospira and Leptospirosis. Melbourne: MediSci.

4. Katz AR, Manea SJ, Sasaki DM, 1991. Leptospirosis on Kauai: investigation of a common source waterborne outbreak. Am J Public Health 81: 1310-1312.

5. Center for Disease Control and Prevention, 1997. Outbreak of leptospirosis among white-water rafters-Costa Rica, 1996. MMWR Morb Mortal Wkly Rep 46: 577-579.

6. Sanders EJ, Rigau-Perez JG, Smits HL, Deseda CC, Vorndam VA, Aye T, Spiegel RA, Weyant RS, Bragg SL, 1999. Increase of leptospirosis in dengue-negative patients after a hurricane in Puerto Rico in 1996. Am J Trop Med Hyg 61: 399-404.

7. Pan American Health Organization, 1998. Impact of Hurricane Mitch in Central America. Bol Epidemiol 19: 1-13.

8. Center for Disease Control and Prevention, 1998. Outbreak of acute febrile illness among athletes participating in triathlonsWisconsin and Illinois, 1998 (published erratum appears in MMWR Morb Mortal Wkly Rep 47: 619). MMWR Morb Mortal Wkly Rep 47: 585-588.

9. Center for Disease Control and Prevention, 2000. Leptospirosis outbreak in Eco Challenge 2000 participants. Commun Dis Rep CDR Wkly 10: 341.

10. Gonçalves A, Santino Filho F, Duarte F, 1967. Weil's disease. Bol Cent Est Hosp Serv Est 19: 147-167.

11. Caldas EM, Costa E, Sampaio MB, 1978. Leptospirosis in Salvador (Brazil). Clinical and laboratory aspects. Rev Inst Med Trop Sao Paulo 20: 164-176.

12. Correa MO, 1975. Human leptospirosis in Brazil. Int J Zoonoses 2: $1-9$.

13. Sakata EE, Yasuda PH, Romero EC, Silva MV, Lomar AV, 1992. The serovars of Leptospira interrogans isolated from cases of human leptospirosis in Sao Paulo, Brazil. Rev Inst Med Trop Sao Paulo 34: 217-221.
14. Ko AI, Galvao Reis M, Ribeiro Dourado CM, Johnson WD Jr, Riley LW, 1999. Urban epidemic of severe leptospirosis in Brazil. Salvador Leptospirosis Study Group. Lancet 354: 820825.

15. Lomar AV, Diament D, Torres JR, 2000. Leptospirosis in Latin America. Infect Dis Clin North Am 14: 23-39.

16. Nicodemo AC, Duarte MI, Alves VA, Takakura CF, Santos RT, Nicodemo EL, 1997. Lung lesions in human leptospirosis: microscopic, immunohistochemical, and ultrastructural features related to thrombocytopenia. Am J Trop Med Hyg 56: 181187.

17. Marotto PC, Nascimento CM, Eluf-Neto J, Marotto MS, Andrade L, Sztajnbok J, Seguro AC, 1999. Acute lung injury in leptospirosis: clinical and laboratory features, outcome, and factors associated with mortality. Clin Infect Dis 29: 1561-1563.

18. Goncalves AJ, de Carvalho JE, Guedes e Silva JB, Rozembaum R, Vieira AR, 1992. Hemoptysis and the adult respiratory distress syndrome as the causes of death in leptospirosis. Changes in the clinical and anatomicopathological patterns. Rev Soc Bras Med Trop 25: 261-270.

19. Barcellos C, Chagastelles Sabroza P, 2000. Socio-environmental determinants of theleptospirosis outbreak of 1996 in western Rio de Janeiro: a geographical approach. Int J Environ Health Res 10: 301-313.

20. Barcellos C, Sabroza PC, 2001. The place behind the case: leptospirosis, risks, and associated environmental conditions in a flood-related outbreak in Rio de Janeiro. Cad Saude Publica 17 (Suppl): 59-67.

21. Thiermann AB, 1977. Incidence of leptospirosis in the Detroit rat population. Am J Trop Med Hyg 26: 970-974.

22. Thiermann AB, Frank RR, 1980. Human leptospirosis in Detroit and the role of rats as chronic carriers. Int $J$ Zoonoses 7: 62-72.

23. Vinetz JM, Glass GE, Flexner CE, Mueller P, Kaslow DC, 1996. Sporadic urban leptospirosis. Ann Intern Med 125: 794-798.

24. Childs JE, Schwartz BS, Ksiazek TG, Graham RR, LeDuc JW, Glass GE, 1992. Risk factors associated with antibodies to leptospires in inner-city residents of Baltimore: a protective role for cats. Am J Public Health 82: 597-599.

25. Terpstra WJ, Ligthart GS, Schoone GJ, 1985. ELISA for the detection of specific IgM and IgG in human leptospirosis. $J$ Gen Microbiol 131: 377-385.

26. Adler B, Murphy AM, Locarnini SA, Faine S, 1980. Detection of specific anti-leptospiral immunoglobulins $\mathrm{M}$ and $\mathrm{G}$ in human serum by solid-phase enzyme-linked immunosorbent assay. $J$ Clin Microbiol 11: 452-457.

27. Instituto Brasileiro de Geografia e Estatística, 1996. Anuário Estatístico do Brasil. Rio de Janeiro: Instituto Brasileiro de Geografia e Estatística.

28. Pereira MM, Matsuo MG, Bauab AR, Vasconcelles SA, Morães ZM, Baranton G, Saint Giron I, 2001. A clonal subpopulation of Leptospira interrogans sensu stricto is the major cause of leptospirosis outbreaks in Brazil. J Clin Microbiol 38: 450-452.

29. Kupek E, de Sousa Santos Faversani MC, de Souza Philippi JM, 2000. The relationship between rainfall and human leptospirosis in Florianopolis, Brazil, 1991-1996. Braz J Infect Dis 4: 131-134.

30. World Bank, 2000. World Development Report 1999/2000. Oxford: Oxford University Press. 